\title{
Analysis of Residual Solvents in Annatto Extracts Using a Static Headspace Gas Chromatography Method
}

\author{
Yusai Ito ${ }^{*}$, Kyoko Ishizuki, Wakana Sekiguchi, Atsuko Tada, Takumi Akiyama, Kyoko Sato, \\ Takeshi Yamazaki, Hiroshi Akiyama \\ Division of Food Additives, National Institute of Health Sciences, Tokyo, Japan \\ Email: *yuito@nihs.go.jp
}

Received July 27, 2012; revised August 27, 2012; accepted September 3, 2012

\begin{abstract}
An analytical method for the quantification of residual solvents in annatto extracts, natural food colorants, was established using a static headspace gas chromatography (HSGC) coupled with a flame ionization detector (FID). As a sample diluent in a headspace sampling, dimethylformamide (DMF) was selected owing to its high capacity for dissolving both bixin-based and norbixin-based annatto extracts. The quantification of residual solvents was performed using the external standard method. The linearity of the calibration curves was assured with relative coefficients $\left(R^{2}\right)$ that were greater than 0.999 . The recoveries of all standard solvents spiked in the annatto extracts were in the range from $95.1 \%$ to $107.1 \%$ to verify the accuracy and the relative standard deviation $(\mathrm{RSD} \%)$ values $(\mathrm{n}=3)$ were in the range from $0.57 \%$ to $3.31 \%$. The quantification limits (QL) were sufficiently lower than the limits specified by Joint FAO/WHO Expert Committee on Food Additives (JECFA). With the established HSGC method, six residual solvents (methanol, ethanol, 2-propanol, acetone, ethyl acetate, and hexane) in 23 commercial annatto-extract products that consist of seven bixin-based and 16 norbixin-based products were quantified. The levels of residual ethyl acetate and hexane in all products were lower than the specified limits of JECFA. However, three samples of bixin-based products showed higher levels of residual 2-propanol (approximately 313.9 - $427.7 \mathrm{ppm}$ ) than the specified limit. Other bixin products also showed higher concentrations of residual methanol (approximately 166.6 - $394.7 \mathrm{ppm}$ ) and residual acetone (approximately $75.2-179.8 \mathrm{ppm}$ ) than the limits of JECFA. In the case of norbixin-based products, nine samples showed higher levels of residual acetone (approximately $42.6-139.5 \mathrm{ppm}$ ) than the limits of JECFA. This is the first survey of residual solvents in annatto extracts using the validated HSGC method.
\end{abstract}

Keywords: Annatto Extracts; Bixin; Norbixin; Headspace Gas Chromatography; Residual Solvents

\section{Introduction}

Annatto extracts are natural yellowish-orange colorants prepared from the seeds of the tropical tree Bixa orellana L. [1]. Annatto extracts have good heat stability during food processing and have been used in many countries to give a yellow-to-red color to foods, especially dairy products such as butter and cheese [1]. The principle pigments of annatto extracts are apocarotenoids, bixin, and norbixin [2] (Figure 1). Bixin is a major natural carotenoid contained in the outer layer of the seed and is a monomethylester of norbixin, a polyenedicarboxylic acid (Figure 1). Bixin is lipophilic in nature and therefore highly insoluble in water. Therefore, crude extracts containing bixin are often hydrolyzed with an alkali to prepare norbixin in order to increase the water solubility of the pigments $[1,2]$. The salts of norbixin obtained by alkali hydrolysis are soluble in water, however, the protonated form of

"Corresponding author. norbixin formed after acid-precipitation purification becomes insoluble (Figure 2).

In 2007, the 39th Codex Committee on Food Additives (CCFA) divided annatto extracts into two classes on the basis of the principle pigments: bixin-based (INS No.160b (1)) and norbixin-based (INS No.160b (2)) [3]. In contrast, in the previous year, the 67th Joint FAO/WHO Expert Committee on Food Additives (JECFA) proposed to classify annatto extracts into five categories on the basis of the manufacturing process in addition to the principle

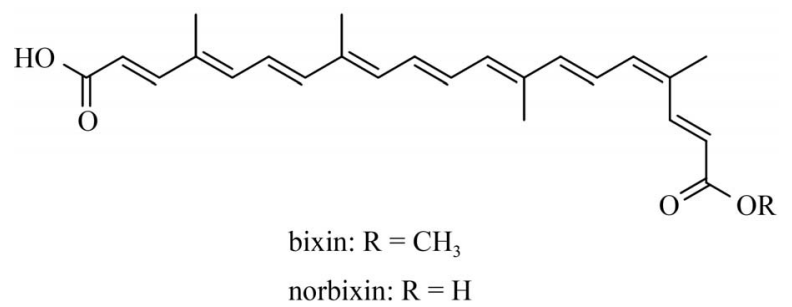

Figure 1. Structures of bixin and norbixin. 

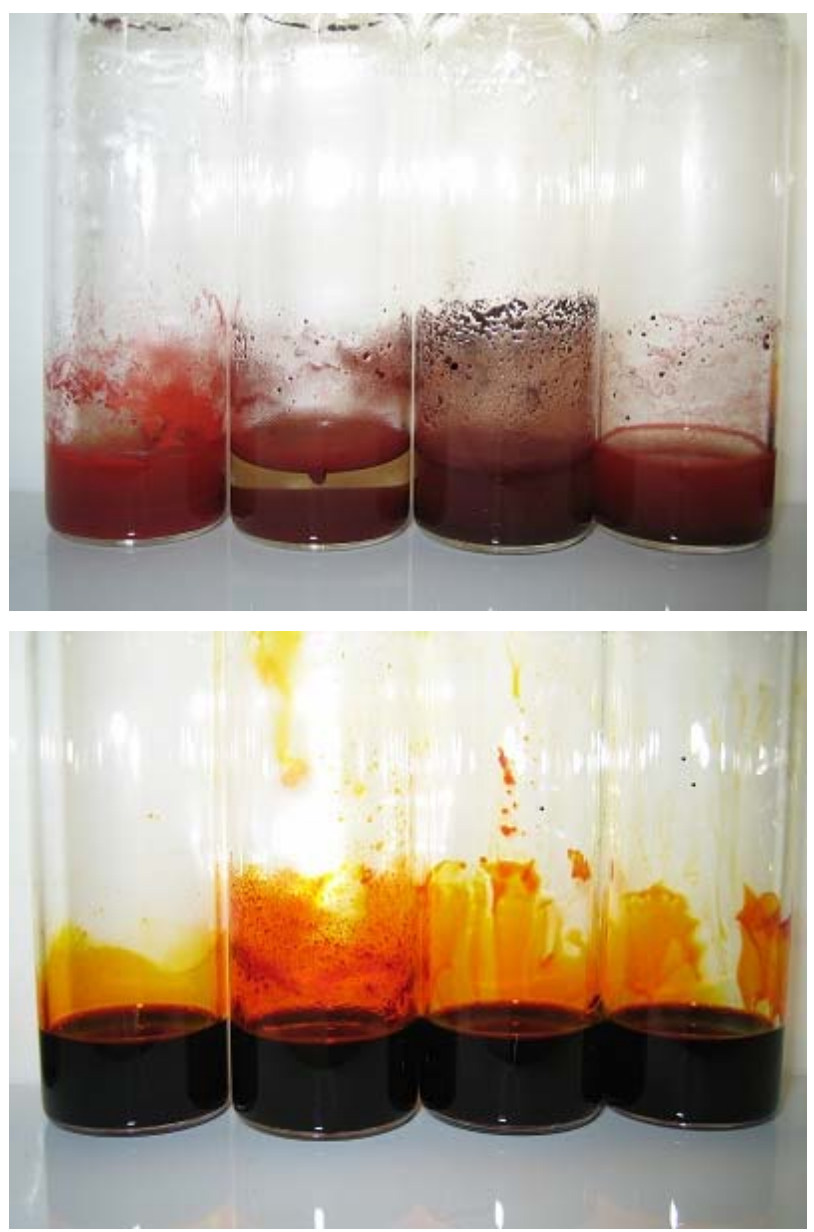

Figure 2. Solubility of annatto extracts in water (upper) and DMF (bottom). The two left bottles are bixin-based products (bix3 and bix4), and the two right bottles are norbixin-based products (nbx4 and nbx8).

pigments [4]: Annatto B (solvent-extracted bixin), Annatto E (aqueous-processed bixin), Annatto C (solvent-extracted norbixin), Annatto F (alkali-processed norbixin, acid precipitated), and Annatto G (alkali-processed norbixin, not acid precipitated). For the production of solvent-extracted bixin and norbixin, i.e. Annatto $\mathrm{B}$ and $\mathrm{C}$, respectively, JECFA permitted the use of six food grade solvents as the extraction solvent: methanol, ethanol, 2-propanol, acetone, ethyl acetate, and hexane, and specified residue limits for each solvent in the final products (Table 1) [4]. The European Food Safety Authority (EFSA) also specified limits for four residual solvents (methanol, acetone, hexane, and dichloromethane) against solvent-extracted bixin and norbixin (Table 1) [5]. The Code of Federal Regulations (CFR) of the United States does not classify annatto extracts, but states that annatto extracts should contain no more than six solvents (methanol, 2-propanol, acetone, hexane, dichloromethane, and trichloroethylene), residues of which are permitted in the corresponding spice oleoresins (Table 1) [6]. In Japan, annatto extracts are allowed to be used
Table 1. Specified limits (ppm) of residual solvents in JECFA, EU, and USA guidelines.

\begin{tabular}{lccc}
\hline Solvent & JECFA & EU & USA \\
\hline Methanol & 50 & ${ }^{*} 50$ & 50 \\
Ethanol & ${ }^{*} 50$ & - & - \\
2-Propanol & ${ }^{*} 50$ & - & 50 \\
Acetone & 30 & ${ }^{*} 50$ & 30 \\
Hexane & 25 & ${ }^{*} 50$ & 25 \\
Ethyl acetate & $* 50$ & - & - \\
Dichloromethane & - & 10 & $* 30$ \\
Trichloroethylene & - & - & $* 30$
\end{tabular}

${ }^{*}$ Individually or in combination.

as one of the existing food additives, but have not yet been listed in the Japanese Standards of Food Additives. Therefore, specified limits of residual solvents are also not established.

JECFA designated static headspace gas chromatography (HSGC) with a flame ionization detector (FID) as the general analytical method for the determination of residual solvents in food additives [7]. The static headspace (HS) sampling method has more appropriate sensitivity than the direct injection method because it can clearly separate volatile analytes from the sample matrix and effectively concentrate them. Therefore, this method results in less complex sample preparation, decreased instrument contamination, and increased gas chromatography (GC) column life. The HS sampling process is based on thermostatic partitioning of volatile compounds between the sample diluent and the gas phase in a sealed vial. Therefore, the selection of the sample diluent is a critical factor affecting the precision of the HSGC analysis. A good sample diluent for HS samplings should have a high stability, high boiling point, and high capability for dissolving large amount of samples [8]. In the general HSGC analytical method of JECFA specification, two solvents are listed as sample diluents: one is water (Method I) and the other is methanol (Method II) [7]. Water is a good diluent for HSGC because it offers a very low partition coefficient for analytes and has a low vapor pressure. However, annatto extracts (bixin and the protonated form of norbixin) are insoluble in water as described above (Figure 2). Although annatto extracts are soluble in methanol, residual methanol in the sample cannot be determined if methanol is used as the diluent. Consequently, the general JECFA method is inapplicable for annatto extracts.

The US Pharmacopeia (USP) General Chapter Residual solvents (467), based on the European Pharmacopeia (EP) procedure [9], presented an HSGC method for testing procedures for residual solvents in pharmaceuticals. Interestingly, the procedures can be divided into two categories on the basis of the solubility of the samples: water-soluble materials and water-insoluble materials $[10,11]$. 
For the water-insoluble procedure, polar organic solvents, such as dimethyl sulfoxide (DMSO) and dimethylformamide (DMF), are designated as the sample diluent. These polar organic solvents have a higher boiling point than water and a high capacity for dissolving a wide range of organic substances. The HSGC method using DMF as the sample diluent has a higher precision than when water is used in the quantification of residual solvents in drug substances [12]. In the JECFA specification of bixin-based products, DMF is designated as the solvent to dissolve and dilute samples in high-performance liquid chromatography (HPLC) analysis for impurities [9]. Therefore, we thought that DMF could become a suitable sample diluent for HSGC analysis of annatto extracts.

There are a few reports on the determination of residual solvents in annatto extracts [13-15]. In these reports, some samples showed high levels of residual methanol. However, most samples analyzed in these reports were not technical products (powder form) but liquid preparations. In addition, the principle pigments of the samples were not described. To our best knowledge, there is no information about residual solvents in annatto extracts after the specification was issued by the $67^{\text {th }}$ JECFA. In order to secure the safety and assure good manufacture practices (GMP) of commercial food additives, a precise quantification of residual solvents is essential. In the present study, we first developed a reliable and analytical method using HSGC for quantification of residual solvents in annatto extracts, and then precisely determined the levels of six residual solvents specified by JECFA in 23 commercial annatto extracts, including both bixin-based and norbixin-based products.

\section{Experimental}

\subsection{Samples}

Twenty-three commercial products containing annatto extracts were collected from Japanese food additives manufacturers in 2011. The samples consisted of six bixin-based products (bix 1-6) and 17 norbixin-based products (nbx117). All samples were red or reddish purple powder and were stored at $-20^{\circ} \mathrm{C}$ until analyzed.

\subsection{Chemical Reagents}

Organic solvents (methanol, ethanol, 2-propanol, acetone, ethyl acetate, and hexane) with $\geq 98 \%$ purity were purchased from Wako Pure Chemical Industries, Ltd (Osaka, Japan). DMF was used for residual solvent analyses and purchased from Kanto Chemical. Co., Inc. (Tokyo, Japan). Water was deionized using a Milli-Q water purification system (Millipore, Bedford, MA).

\subsection{Sample Preparation}

A quantity $(200 \mathrm{mg})$ of each annatto extract sample was accurately weighed in an Agilent $20 \mathrm{~mL}$ HS sample vial and DMF $(2.5 \mathrm{~mL})$ was added. The vial was immediately capped and sealed with a Teflon-lined septum and aluminum crimp cap, and then mixed thoroughly until the entire sample was dissolved. Then, the capped vial was placed in the oven of the HS sampler. All samples were prepared in triplicate.

\subsection{Standard Solutions and Calibration Curves}

A stock standard solution for each solvent was prepared as follows. Each $250 \mu \mathrm{L}$ of methanol, ethanol, 2-propanol, and ethyl acetate, and each $150 \mu \mathrm{L}$ of acetone and hexane was pipetted into a volumetric flask $(20 \mathrm{~mL})$ into which DMF $(10 \mathrm{~mL})$ had previously been added. The flasks were weighed to within $0.01 \mathrm{mg}$ and then filled to capacity with DMF. A stock standard mixture solution was prepared by placing each stock standard solution $(3.0 \mathrm{~mL})$ in a volumetric flask $(20 \mathrm{~mL})$ and filling the flask to capacity with DMF. Standard mixture solutions used for the calibration curve were prepared by sequentially diluting the stock standard mixture solution with DMF to seven concentration levels.

For the HSGC analysis, the standard mixture solution $(0.1 \mathrm{~mL})$ was pipetted into an Agilent $20 \mathrm{~mL}$ headspace HS sample vial and DMF $(2.4 \mathrm{~mL})$ was added to the vial. The vial was immediately capped and sealed as mentioned above. The samples were prepared in triplicate. To establish calibration equations, the mean peak areas $(n=3)$ of standard solvents observed by HSGC analysis were plotted against concentration. External calibration curves were established over seven datapoints covering a concentration range of approximately $1.0-700 \mathrm{ppm}$ for methanol, ethanol, 2-propanol, and ethyl acetate and approximately 0.5 - $350 \mathrm{ppm}$ for acetone and hexane. All the solvent concentrations were calculated on the basis of the 200 $\mathrm{mg}$ annatto extracts being dissolved in $2.5 \mathrm{~mL}$ of DMF. The final concentration of each standard solution used for the calibration curve is shown in Table 2.

\subsection{Headspace Gas Chromatography Procedure}

An Agilent 6890 N GC equipped with an FID and a 7694

Table 2. Retention time and linearity of six standards solvents.

\begin{tabular}{lccc}
\hline Solvent & RT (min) & Range (ppm) & $R^{2}$ \\
\hline Methanol & 5.148 & $0.9-679.2$ & 0.9997 \\
Ethanol & 6.452 & $1.0-726.6$ & 0.9992 \\
2-Propanol & 7.542 & $1.0-740.5$ & 0.9996 \\
Acetone & 8.068 & $0.5-369.5$ & 0.9998 \\
Hexane & 9.608 & $0.4-327.8$ & 0.9999 \\
Ethyl acetate & 12.713 & $1.0-717.4$ & 0.9998 \\
\hline
\end{tabular}


$\mathrm{K}$-special HS sampler was used for the experiments. The GC column was a GL Sciences AQUATIC-2 (25\% phenyl $/ 75 \%$ methyl polysiloxane) — fused silica capillary column: length, $60 \mathrm{~m}$; internal diameter, $0.25 \mathrm{~mm}$; film thickness, 1.40 mm (Part No. 123-1334, Serial No. US1613334$\mathrm{H})$. The initial temperature of the column oven was $40^{\circ} \mathrm{C}$, and this was maintained for $5 \mathrm{~min}$, then raised at a rate of $4^{\circ} \mathrm{C} / \mathrm{min}$ to $92^{\circ} \mathrm{C}$ and maintained for $2 \mathrm{~min}$, and then raised at a rate of $40^{\circ} \mathrm{C} / \mathrm{min}$ to $230^{\circ} \mathrm{C}$. The injection temperature was $250^{\circ} \mathrm{C}$, and the FID detector temperature was $260^{\circ} \mathrm{C}$. Helium at $205 \mathrm{kPa}$ was used as the carrier gas (constant flow, $1.8 \mathrm{~mL} / \mathrm{min}$ ) and the split ratio was $25: 1$. The headspace HS was sampled as follows: the vial was maintained at $60^{\circ} \mathrm{C}$ for $20 \mathrm{~min}$ with continuous agitation. The size of injection loop was $3 \mathrm{~mL}$. The needle temperature was $100^{\circ} \mathrm{C}$ and the transfer line temperature was $120^{\circ} \mathrm{C}$.

\subsection{Recovery}

Recovery rates of standard solutions for three selected samples (bix 1, bix4, and nbx8) were calculated using the standard addition method. The standard mixture solution spiked in the sample was individually prepared as follows. Bix 1: stock standard solutions of methanol $(0.2 \mathrm{~mL})$, ethanol $(0.2 \mathrm{~mL}), 2$-propanol $(1.4 \mathrm{~mL})$, acetone $(0.4 \mathrm{~mL})$, ethyl acetate $(0.2 \mathrm{~mL})$, and hexane $(0.2 \mathrm{~mL})$ were pipetted into a volumetric flask $(20 \mathrm{~mL})$ and filled with DMF. Bix4: stock solutions of methanol (1.4 mL), ethanol $(0.2$ $\mathrm{mL})$, 2-propanol $(0.2 \mathrm{~mL})$, acetone $(1.4 \mathrm{~mL})$, ethyl acetate $(0.2 \mathrm{~mL})$, and hexane $(0.2 \mathrm{~mL})$ were pipetted into a volumetric flask $(20 \mathrm{~mL})$ and filled with DMF. Nbx8: stock solutions of methanol $(0.2 \mathrm{~mL})$, ethanol $(0.2 \mathrm{~mL})$, 2-propanol $(0.2 \mathrm{~mL})$, acetone $(0.8 \mathrm{~mL})$, ethyl acetate $(0.2$ $\mathrm{mL})$, and hexane $(0.2 \mathrm{~mL})$ were pipette in a volumetric flask $(20 \mathrm{~mL})$ and filled with DMF. The final concentration of each spiked standard solution was shown in Table 3. The samples $(200 \mathrm{mg})$ were separately weighed in a HS vial $(20 \mathrm{~mL})$, dissolved in DMF $(2.4 \mathrm{~mL})$, and spiked with the standard mixture solution $(0.1 \mathrm{~mL})$ prepared for each sample. Quantitative analysis was performed by the

Table 3. Quantification limit (QL) and detection limit (DL) and precision of six solvents.

\begin{tabular}{lcccc}
\hline Solvent & QL $(\mathrm{ppm})$ & $\mathrm{DL}(\mathrm{ppm})$ & $\begin{array}{c}\text { Precision at } \\
\mathrm{WL}^{*} \\
(\mathrm{RSD} \%, \mathrm{n}=3)\end{array}$ & $\begin{array}{c}\text { Precision at } \\
\mathrm{LL}^{* *}\end{array}$ \\
\hline Methanol & 12.93 & 3.53 & 2.79 & 8.98 \\
Ethanol & 13.22 & 3.61 & 2.69 & 4.61 \\
2-Propanol & 14.25 & 3.98 & 3.31 & 4.14 \\
Acetone & 3.64 & 0.92 & 0.82 & 4.79 \\
Hexane & 0.30 & 0.01 & 0.57 & 0.75 \\
Ethyl acetate & 6.23 & 1.62 & 0.68 & 4.89 \\
\hline
\end{tabular}

${ }^{*}$ Working concentration level $(20-50 \mathrm{ppm}) ;{ }^{* *}$ Low concentration level (4 $10 \mathrm{ppm})$.
HSGC procedure as described above. The recovery rate was calculated by comparing the amount of standard in the spiked sample with the amount in the non-spiked annatto extract sample (control). Each analysis was performed in triplicate.

\section{Results and Discussion}

\subsection{Headspace Gas Chromatography Method}

As the sample diluent for HSGC analysis, DMF was selected owing its high boiling point and high capacity for dissolving organic compounds. As expected, DMF was able to dissolve both bixin-based and norbixin-based products, while water, designated as a sample diluent by JECFA, was unable to dissolve either product (Figure 2). The equilibration temperature for HS sampling was set at $60^{\circ} \mathrm{C}$, because this is the JECFA-recommended temperature for the general HSGC method, and it was reported that bixin gradually degrades to several products at temperatures above $70^{\circ} \mathrm{C}$ [16]. The equilibration time for $\mathrm{HS}$ sampling was determined as $20 \mathrm{~min}$ on the basis of the saturation of peak areas of standard solutions on the gas chromatogram (data not shown). The established HSGC procedure using a capillary column AQUATIC-2 gave a good separation of six standard solvent peaks (methanol, ethanol, 2-propanol, acetone, ethyl acetate, and hexane) on the chromatogram (Figure 3). Retention time for each solvent is shown in Table 2. To assess linearity, calibration curves of six solvents were constructed over a range of seven concentrations using standard mixture solutions. Good linearity was achieved over the concentration ranges of approximately 1.0 - 700 ppm for methanol, ethanol, 2-propanol, and ethyl acetate, and approximately 0.5 - 350 ppm for acetone and hexane (Table 2). The regression coefficients $\left(R^{2}\right)$ for the curves of six solvents range from 0.9992 to 0.9999 (Table 2). The sensitivity of the HSGC method is presented as QL with a signal-noise ratio of 10:1, and detection limit (DL) with a signal-noise ratio of $3: 1$. The QL values of methanol, ethanol, and 2-propanol are evaluated in the range from 12.93 to $14.25 \mathrm{ppm}$ and the values of other solvents ranged from 0.30 to $6.23 \mathrm{ppm}$ (Table 3). Because the QL values of all solvents are satisfactorily lower than the specified limits required by the JECFA guideline, the results demonstrate that the established HSGC method is sufficiently sensitive for the quantification of residues of six solvents in annatto extracts. To assess the accuracy of the method, recovery rates for six solvents were calculated using the spike of a standard solution mixture to three samples (bix1, bix4, and nbx8). Each concentration of the spiked solvent standard was selected on the basis of the specified limits of JECFA (Table 4). When the concentrations of the residual solvent measured using the HSGC method significantly exceeded the specified limits, the concentrations of spiked solvent 
standards were selected on the basis of the measured concentrations, such as acetone (49.3 ppm) and 2-propanol (345.6 ppm) in bix 1, methanol (317.0 ppm) and acetone (172.4 ppm) in bix4, and acetone (98.5 ppm) in nbx 8 (Table 4). When the standard mixture solution was spiked at defined amounts in each sample prior to quantitative analysis, the recovery rates of the spiked standards in all samples were within the range of $95.0 \%-109.7 \%$ during HSGC analysis (Table 4). Good recoveries clearly revealed that interference from the sample matrix should not have a significant impact on this HSGC method. Therefore, we considered that the external standard method was applied

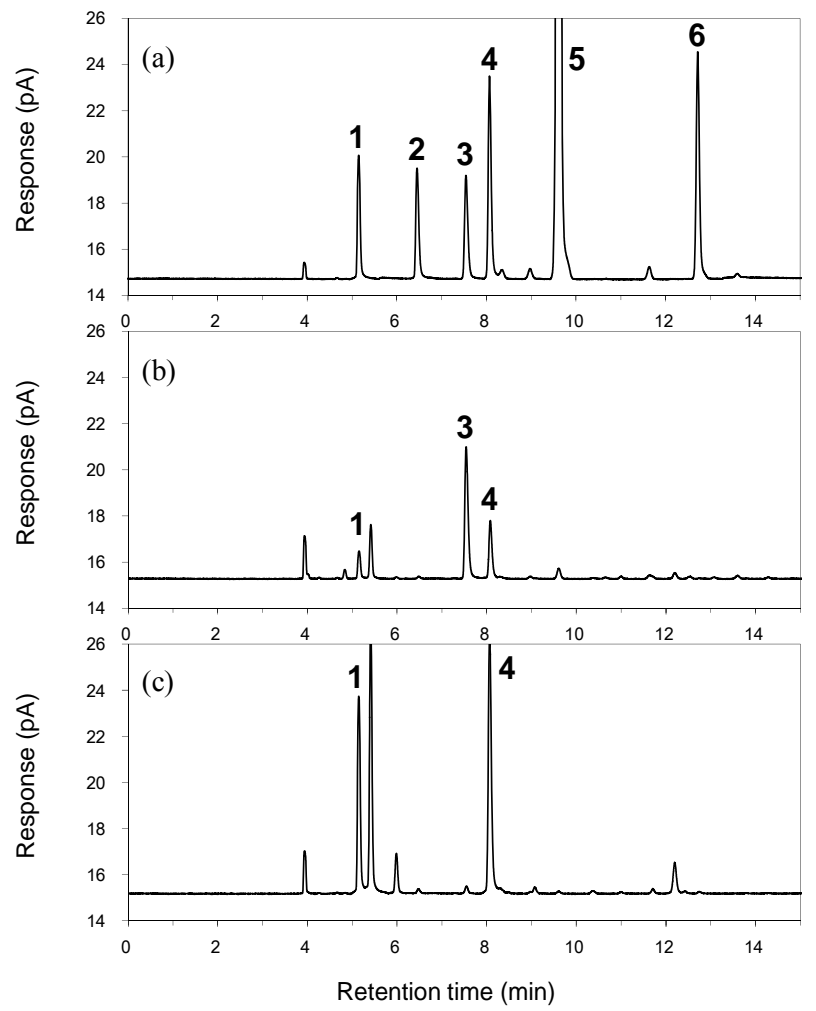

Figure 3. HSGC charts of (a) standard mixture; (b) bix1; and (c) bix4. Peak identities and concentrations in the standard mixture (a) are as follows: methanol (1, 270 ppm), ethanol (2, $290 \mathrm{ppm}), 2$-propanol (3, $300 \mathrm{ppm})$, acetone (4, $150 \mathrm{ppm})$, hexane (5, $130 \mathrm{ppm})$, ethyl acetate (6, $290 \mathrm{ppm})$. for the determination of residual solvents in annatto extracts. The precision of the method was tested by multiple injection $(\mathrm{n}=3)$ of the standard mixture at both working-concentration level (WL, 20 - $50 \mathrm{ppm}$ ) on the basis of the specified limits of JECFA, and a lower concentration level (LL, $4-10 \mathrm{ppm}$ ) on the basis of the QL values. The RSD values were in the range $0.57 \%-3.31 \%$ at the WL and $0.75 \%-8.98 \%$ at the LL (Table 3).

These results clearly demonstrate that the developed HSGC method has acceptable linearity, accuracy, and precision, and is a reliable method for the accurate quantitative determination of residual solvents in annatto extracts.

\subsection{Residual Solvents in Bixin-Based Products}

Using the established HSGC method, the residual solvents in 23 annatto extract products were precisely quantified (Table 5). Representative chromatograms of bixin-based products (bix 1 and bix4) are shown in Figure 3. In six bixin-based products, three samples (bix 1-3) showed high concentrations of residual 2-propanol (approximately 313.9 - $427.7 \mathrm{ppm}$ ), which were much higher than the specified limit of JECFA (50 ppm). Because these samples were produced by the same manufacture, we presumed that the detected 2-propanol was likely to be a residue of the solvent used in the manufacturing process. Although other bixin-based samples (bix4-6) showed a lower concentration of residual 2-propanol, concentrations of residual methanol (approximately 112.8 - 383.5 ppm) and residual acetone (approximately $73.4-180.1 \mathrm{ppm}$ ) higher than the JECFA limits (50 and 30 ppm, respectively) were quantified. These may also be residue of solvents used in the manufacturing process, although the methanol might be generated by hydrolysis of the methylester in bixin during storage. Scotter et al. reported the powdered bixin is more unstable than oleoresin bixin and gradually degrades in complex reactions, even in dark and cold conditions [1]. In fact, bix 4 was stored for a few years before used in this study. In the case of the sample containing $75 \%$ (w/w) bixin, we estimated that the degradation of only $0.16 \%(\mathrm{w} / \mathrm{w})$ of bixin could generate $100 \mathrm{ppm}$ of methanol in the sample. As a similar example, Sato et al. reported that a natural food colorant, gardenia blue, which

Table 4. Recoveries of six solvents spiked in three samples.

\begin{tabular}{|c|c|c|c|c|c|c|}
\hline \multirow{2}{*}{$\begin{array}{l}\text { Sample } \\
\text { Solvent }\end{array}$} & \multicolumn{2}{|c|}{ bix 1} & \multicolumn{2}{|c|}{ bix4 } & \multicolumn{2}{|c|}{ nbx8 } \\
\hline & Spiked (ppm) & Recovery (\%) & Spiked (ppm) & Recovery (\%) & Spiked (ppm) & Recovery (\%) \\
\hline Methanol & 45.3 & 96.5 & 317.0 & 102.8 & 45.3 & 102.7 \\
\hline Ethanol & 48.4 & 95.0 & 48.4 & 100.2 & 48.4 & 101.2 \\
\hline 2-Propanol & 345.6 & 101.6 & 49.4 & 100.2 & 49.4 & 102.6 \\
\hline Acetone & 49.3 & 101.9 & 172.4 & 104.1 & 98.5 & 98.4 \\
\hline Hexane & 21.9 & 97.3 & 21.9 & 98.2 & 21.9 & 100.1 \\
\hline Ethyl acetate & 47.8 & 99.3 & 47.8 & 105.2 & 47.8 & 103.0 \\
\hline
\end{tabular}


Table 5. Concentrations of residual solvents in $\mathbf{2 3}$ commercial products.

\begin{tabular}{|c|c|c|c|c|c|c|c|c|c|c|c|c|c|}
\hline \multirow{3}{*}{ Type } & \multirow{3}{*}{$\frac{\text { No. }}{\text { bix1 }}$} & \multicolumn{12}{|c|}{ Concentrations of residual solvents (ppm) and RSD (\%) } \\
\hline & & \multicolumn{2}{|c|}{ Methanol } & \multicolumn{2}{|c|}{ Ethanol } & \multicolumn{2}{|c|}{ 2-Propanol } & \multicolumn{2}{|c|}{ Acetone } & \multicolumn{2}{|c|}{ Hexane } & \multicolumn{2}{|c|}{ Ethyl acetate } \\
\hline & & 49.7 & $(0.4)$ & nd & & 369.3 & $(1.2)$ & 44.0 & $(2.0)$ & 0.6 & $(1.4)$ & nd & \\
\hline \multirow{5}{*}{ Bixin } & bix 2 & 38.2 & $(5.0)$ & nd & & 316.2 & $(1.0)$ & 28.2 & (3.7) & 0.7 & $(9.9)$ & nd & \\
\hline & bix 3 & 35.0 & $(2.9)$ & nd & & 438.4 & $(2.8)$ & 27.5 & $(1.7)$ & nd & & nd & \\
\hline & bix 4 & 394.5 & (4.7) & nd & & nd & & 179.8 & (5.0) & nd & & nd & \\
\hline & bix 5 & 119.4 & (1.1) & nd & & nd & & 95.7 & (1.8) & nd & & nd & \\
\hline & bix6 & 166.0 & $(0.7)$ & nd & & nd & & 75.2 & $(0.2)$ & nd & & nd & \\
\hline \multirow{17}{*}{ Norbixin } & nbx1 & nd & & nd & & nd & & 27.1 & (2.4) & nd & & nd & \\
\hline & $\mathrm{nbx} 2$ & nd & & nd & & nd & & 23.4 & $(2.2)$ & nd & & nd & \\
\hline & nbx3 & nd & & nd & & nd & & 26.8 & $(4.2)$ & nd & & nd & \\
\hline & nbx4 & 41.1 & $(1.5)$ & nd & & nd & & 106.0 & $(0.8)$ & nd & & nd & \\
\hline & nbx5 & nd & & nd & & nd & & 102.7 & (1.8) & nd & & nd & \\
\hline & nbx6 & nd & & nd & & nd & & 45.0 & (3.1) & nd & & nd & \\
\hline & nbx7 & 432.9 & (1.9) & 4375 & $(1.0)$ & nd & & 36.0 & (1.8) & nd & & 11.5 & (2.5) \\
\hline & nbx 8 & 23.8 & $(2.0)$ & nd & & nd & & 98.8 & (2.0) & nd & & nd & \\
\hline & nbx9 & 25.6 & $(1.2)$ & nd & & nd & & 24.8 & $(0.2)$ & nd & & nd & \\
\hline & nbx 10 & 51.2 & $(0.5)$ & nd & & nd & & 19.3 & $(2.1)$ & nd & & nd & \\
\hline & nbx11 & nd & & nd & & nd & & 44.2 & (4.4) & nd & & nd & \\
\hline & $\mathrm{nbx} 12$ & nd & & nd & & nd & & 33.6 & $(0.8)$ & nd & & nd & \\
\hline & nbx 13 & nd & & nd & & nd & & 25.1 & (2.6) & nd & & nd & \\
\hline & nbx 14 & nd & & nd & & nd & & nd & & nd & & nd & \\
\hline & nbx 15 & nd & & nd & & nd & & nd & & nd & & nd & \\
\hline & nbx 16 & nd & & nd & & nd & & 42.6 & (1.4) & nd & & nd & \\
\hline & nbx17 & nd & & 26.0 & $(0.8)$ & 29.8 & (1.6) & 139.5 & (3.6) & nd & & nd & \\
\hline
\end{tabular}

Data are means for three trials; nd $=$ not determined.

contains methylester structures, showed a high concentration of residual methanol, and suggested that methanol could be generated by spontaneous hydrolysis of the methylester [17]. Based on this knowledge, the concentration limit of residual methanol in gardenia blue is set as $1000 \mathrm{ppm}$ in Japanese Standards of Food Additives. It might be necessary to investigate the generation of methanol by the degradation of bixin during storage. The residue levels of other solvents (ethanol, ethyl acetate, and hexane) were lower than QL in bixin-based samples.

\subsection{Residual Solvents in Norbixin-Based Products}

The represented chromatograms of norbixin-based products were shown in Figure 4 (nbx4, nbx7, and nbx14). In the case of norbixin-based samples, the levels of residual 2-propanol, hexane, and ethyl acetate were lower than the limits of JECFA. However, residual acetone was detected at a higher concentration than the JECFA limit (30 ppm) in nine samples (nbx4, 5, 6, 7, 8, 11, 12, 16, and 17). Although the origin of acetone in the norbixin-based products is not as clear as in the bixin-based products, it should be noted that residual acetone was determined in 15 out of 17 samples examined. Residual methanol in the norbixin-based samples was at a lower level than the specified limit, with the exception of nbx 7 . In nbx7, ethanol was also detected at high concentration (4375 ppm) in addition to residual methanol, suggesting that the results may be caused by imperfect purification in the manufacturing process.

\section{Conclusion}

In this study, a reliable HSGC method using DMF as sample diluent is established for the determination of residual solvents in annatto extracts. With the established method, six residual solvents (methanol, ethanol, 2-propanol, acetone, ethyl acetate, and hexane) specified by JECFA were precisely determined in 23 commercial bixin-based and norbixin-based products. The results revealed that some bixin-based products contained a higher concentration of residual methanol and 2-propanol than the JECFA-specified limit (50 ppm). Furthermore, 13 samples showed a higher concentration of residual acetone than the specified JECFA limit (30 ppm). We would like to note that 

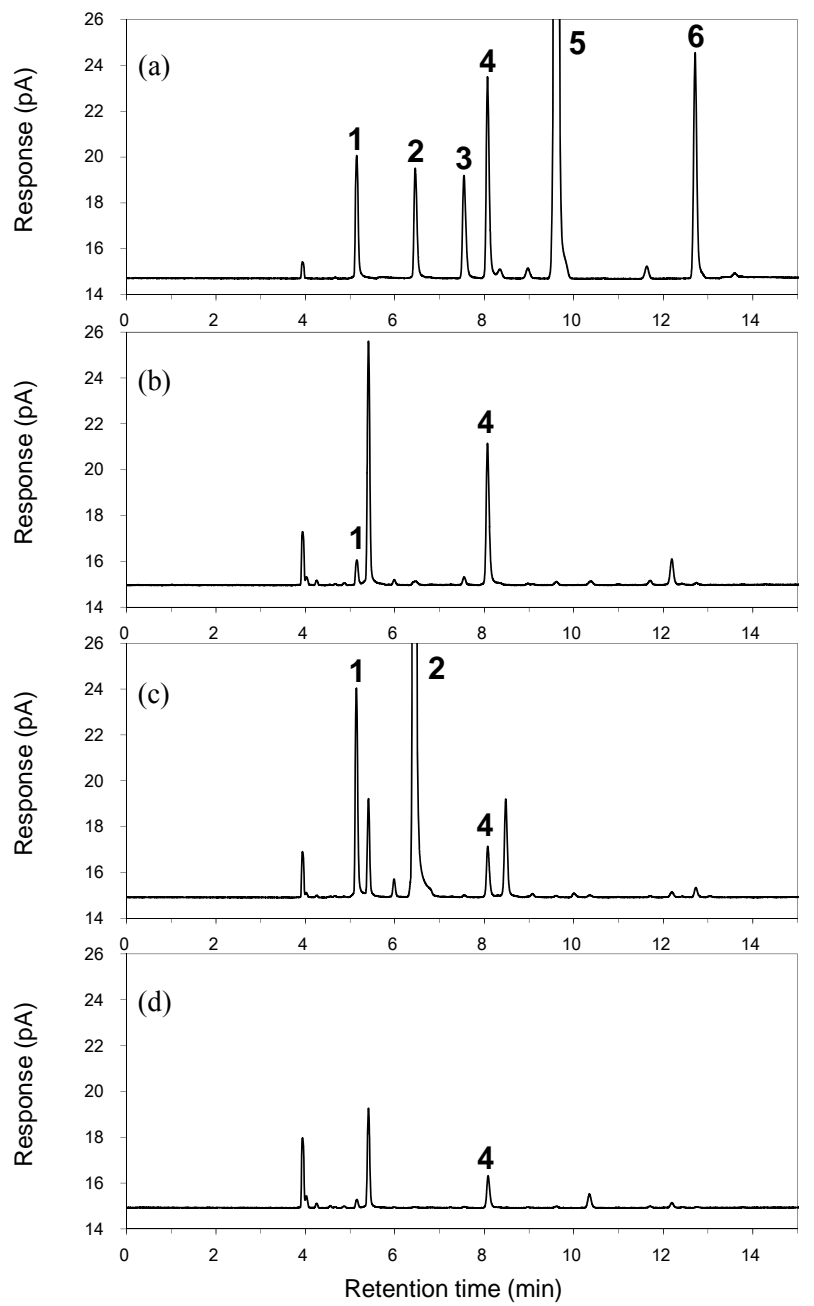

Figure 4. HSGC charts of (a) standard mixture; (b) nbx4; (c) nbx7; and (d) nbx14. Peak identities and concentrations in the standard mixture (a) are the same as in Figure 3.

all products used in this study were imported and were not processed by Japanese manufactures. In short, the findings of this study imply that annatto extracts distributed worldwide also contained a similar level of residual solvents as detected in this study. Based on the results, further investigation on worldwide products and a reevaluation of the current specified limits for residual solvents in annatto extracts is required.

\section{REFERENCES}

[1] M. Scotter, "The Chemistry and Analysis of Annatto Food Colouring: A Review," Food Additives and Contaminants, Vol. 26, No. 8, 2009, pp. 1123-1145. doi:10.1080/02652030902942873

[2] M. J. Scotter, S. A. Thorpe, S. L. Reynolds, L. A. Wilson and P. R. Strutt, "Characterization of the Principal Colouring Components of Annatto Using High Performance Liquid Chromatography with Photodiode-Array Detection," Food Additives and Contaminants, Vol. 119, No. 3,
1994, pp. 301-315. doi:10.1080/02652039409374229

[3] Codex Alimentarius Commission, "Report of the 39th Session of the Codex Committee on Food Additives," Beijing, 24-28 April 2007.

[4] Joint FAO/WHO Expert Committee of Food Additives, "FAO JECFA Monographs 3 Combined Compendium of Food Additive Specification," Vol. 3, Food and Agriculture Organization of the United Nations, Rome, 2006, pp. 3-7.

[5] "Commission Directive 2008/128/EC of December 22, 2008 Laying down Specific Purity Criteria Concerning Colours for Use in Foodstuffs," Official Journal of the European Union, Vol. 6, 2009, pp. 20-63.

[6] "Code of Federal Regulations, Title 21, Part 73 Listing of Color Additives Exempt from Certification, Subpart A Foods, Sec. \$73.30 Annatto Extract,” 2011.

[7] Joint FAO/WHO Expert Committee of Food Additives, "FAO JECFA Monographs 1, Combined Compendium of Food Additive Specification," Vol. 4, Food and Agriculture Organization of the United Nations, Rome, 2006, pp. 87-89.

[8] C. Cheng, S. Liu, B. J. Mueller and Z. Yan, "A Generic Static Headspace Gas Chromatography Method for Determination of Residual Solvents in Drug Substance," Journal of Chromatography A, Vol. 1217, No. 41, 2010, pp. 6413-6421. doi:10.1016/j.chroma.2010.08.016

[9] R. Otero, G. Carrera, J. F. Dulsat, J. L. Fabregas and J. Claramunt, "Static Headspace Gas Chromatographic Method for Quantitative Determination of Residual Solvents in Pharmaceutical Drug Substances According to European Pharmacopoeia Requirements," Journal of Chromatography A, Vol. 1057, No. 1-2, 2004, pp. 193- 201. doi:10.1016/j.chroma.2004.09.023

[10] "US Pharmacopeia 32-National Formulary 27, Residual Solvents <467>," Suppl. 1, Rockville, MD: USP, 2009, p. 3948.

[11] J. L. Belsky, A. J. Ashley, P. A. Bhatt, K. V. Gilbert, H. R. Joyce, C. Pan, H. Pappa and S. Z. Wahab, "Optimization of the Water-Insoluble Procedures for USP General Chapter Residual Solvents <467>," AAPS PharmSciTech, Vol. 2, No. 2, 2010, pp. 994-1004. doi:10.1208/s12249-010-9460-6

[12] S. Klick and A. Sköld, "Validation of a Generic Analytical Procedure for Determination for Residual Solvents in Drug Substances," Journal of Pharmaceutical and Biomedical Analysis, Vol. 36, No. 2, 2004, pp. 401-409. doi:10.1016/i.jpba.2004.06.014

[13] Y. Uematsu, M. Hirokado, K. Hirata, K. Nakajima and M. Karamu, "Determination of Residual Organic Solvent in Natural Color Preparations by Standard Addition HeadSpace Gas Chromatography," Journal of the Food Hygienic Society of Japan, Vol. 34. No. 3, 1993, pp. 232-238. doi:10.3358/shokueishi.34.232

[14] Y. Uematsu, M. Ogimoto, K. Suzuki, J. Kabashima, K. Ito and M. Nakazato, "Survey of Residue Levels of Organic Solvents in 'Existing Food Additives' and Health Food Materials by Head-Space GC," Journal of the Food Hygienic Society of Japan, Vol. 49, No. 5, 2008, pp. 
366-375. doi:10.3358/shokueishi.49.366

[15] Y. Uematsu, K. Hirata, K. Suzuki, K. Iida and K. Kamata, "Survey of Residual Solvents in Natural Food Additives by Standard Addition Head-Space GC," Food Additives and Contaminants, Vol. 19, No. 4, 2002, pp. 335-342. doi:10.1080/02652030110088301

[16] M. J. Scotter, L. A. Wilson, G. P. Appleton and L. Castle, "Analysis of Annatto (Bixa orellana) Food Coloring Formulations. 1. Determination of Coloring Components and
Colored Thermal Degradation Products by High-Performance Liquid Chromatography with Photodiode Array Detection," Journal of Agricultural and Food Chemistry, Vol. 46, No. 3, 1998, pp. 1031-1038. doi:10.1021/jf970063+

[17] K. Sato and T. Maitani, "Determination of Methanol in Gardenia Blue and Gardenia Red," ShokuhinEiseigakuZasshi, Vol. 44, No. 1, 2003, pp. 73-76. doi:10.3358/shokueishi. 44.73 